\title{
EL PRON O QUIPU MAPUCHE SEGÚN FUENTES COLONIALES Y DATOS ARQUEOLÓGICOS: ANTECEDENTES SOBRE SU ORIGEN, USO Y FUNCIÓN
}

\author{
THE PRON, OR MAPUCHE QUIPU, ACCORDING TO \\ COLONIAL RECORDS AND ARCHAEOLOGICAL DATA: ORIGIN, \\ USES AND FUNCTIONS
}

José Manuel Zavala ${ }^{A}$, Dagmar Bachraty ${ }^{B}$ Gertrudis Paỳ̀s ${ }^{c}$

El artículo presenta un balance de los datos existentes en la documentación colonial sobre la presencia del quipu en Chile central y en la Araucanía, y se agrega información proveniente de registros arqueológicos de petroglifos del área andina de Chile central y norte de Neuquén, Argentina. La información etnohistórica y arqueológica permite proponer la presencia del quipu mapuche en tiempos prehispánicos y las referencias coloniales permiten establecer algunos usos y funciones, describir sus componentes y variantes, y su asociación a la guerra. Para el territorio mapuche bajo dominio inca, algunos antecedentes hacen pensar en la asociación del quipu con representaciones petroglíficas ligadas a rutas o sitios mineros.

Palabras clave: Khipu, Püron, Calendario indígena, Guerra mapuche, Sistema de cómputo indígena, Petroglifos, Collasuyo.

This article reviews the evidence in colonial documents on the presence of quipus in central Chile and in Araucania, also including archaeological data from petroglyphs in Andean areas of central Chile and northern Neuquén in Argentina. The ethnohistorical and archaeological information suggests the presence of the Mapuche quipu in pre-Hispanic times. The colonial references enable the identification of some uses and functions, as well as the description of its components and variants, and of its war association. Regarding the Mapuche territory under Inca rule, some records allow to associate this artefact with petroglyph representations linked to mining sites or routes.

Keywords: Khipu, Püron, Indigenous calendar, Mapuche war, Indigenous counting systems, Petroglyphs, Collasuyo.

\section{INTRODUCCIÓN}

La relación entre los mundos prehispánicos mapuche y andino es un tema que se explora desde hace tiempo (Dillehay \& Gordon 1998; Guevara 1908, 1925, 1928; Gusinde 1916; Latcham 1908, 1924, Medina 1882; Uhle 1912) y sobre el cual se han dado pasos que muestran cada vez más influjos de lo andino en lo mapuche o de fenómenos de interdigitación (Bahamondes 2009, Dillehay 2011, Moulian \& Catrileo 2013, Uribe \& Sánchez 2016, Zavala \& Dillehay 2010). ${ }^{1}$

Siguiendo esta ruta temática, nos ha interesado pesquisar el origen y desarrollo de la versión mapuche del principal sistema de registro y comunicación ideográfico andino: el quipu (khipu, en quechua). Nuestra motivación se enmarca en la búsqueda de antecedentes sobre minería aurífera prehispánica de la Araucanía (Zavala et al. 2020), por lo que, estudiar la presencia de este instrumento entre los mapuches -denominado por ellos pron (püron en mapudungun) - cobra sentido en cuanto la expansión inca en Chile estuvo ligada principalmente a la extracción minera (Iribarren \&

A José Manuel Zavala, Departamento de Ciencias Históricas, Facultad de Filosofía y Humanidades, Universidad de Chile, Santiago, Chile. Orcid: 0000-0001-6852-1222.E-mail: josezavala@uchile.cl

B Dagmar Bachraty, Centro de Estudios Culturales Latinoamericanos, Facultad de Filosofía y Humanidades, Universidad de Chile, Santiago, Chile. Orcid: 0000-0002-6020-0579. E-mail: djbachraty@uc.cl

C Gertrudis Payàs, Departamento de Lenguas y Núcleo de Estudios Interétnicos e Interculturales, Universidad Católica de Temuco, Temuco, Chile. Orcid: 0000-0002-9117-8108. E-mail: gpayas@uct.cl 
Wissing 1972, Salazar et al. 2001, Cruz 2013, Raffino et al. 2013).

En este artículo hacemos un balance de los datos existentes en la documentación colonial respecto de la presencia del quipu en Chile central (mapuches del norte) y en la Araucanía ${ }^{2}$ (mapuches del sur). Además, agregamos información proveniente de registros arqueológicos de petroglifos del área andina de Chile central y norte de Neuquén, Argentina. La combinación de información etnohistórica y arqueológica nos permite identificar la presencia prehispánica de este sistema de registro y comunicación por medio de cordeles y nudos. Por otra parte, el análisis de las referencias coloniales hace posible establecer algunos usos y funciones del quipu mapuche, describir sus componentes y variantes, así como su especial desarrollo asociado a la guerra.

Después de revisar las fuentes coloniales publicadas, localizamos catorce textos con referencias a este sistema de registro, con ocurrencias que van desde 1558 hasta 1792. De estos, seleccionamos once con más certeza cronológica para las doce ocurrencias que reportan (tablas 1 y 2). En cuatro ocasiones asignamos una fecha de ocurrencia diferente a la referida en las fuentes: al dato de González de Nájera (1889 [1614]) le atribuimos la fecha del último año de su presencia en Chile, es decir, 1607; la referencia de Núñez de Pineda y Bascuñán (1863 [1673]) proviene de su cautiverio entre los mapuches del año 1629; la descripción de Diego de Rosales (1877-1878 [1674]: 112-113, 52) no puede ser posterior al término de su relato histórico que acaba hacia 1650, y el registro de Stevenson (1825: 50-51) lo dejamos en la fecha asignada por él, es decir, 1792.

Tanto la búsqueda documental como los análisis se facilitaron y apoyaron en trabajos anteriores, principalmente los de Carmen Arellano (2009), Blanco et al. (2015), Jimena Obregón Iturra -aún inédito- (2016), Alberto Trivero (2018) y los clásicos estudios de J. T. Medina (1882) y Ricardo Latcham (1924).

\section{EL QUIPU EN EL MUNDO ANDINO}

Debido a su peculiaridad mnemotécnica, los quipus han sido conocidos como "nudos que hablan". Estos sistemas de registro visual-táctil consistían en hilos de pelo de llama, alpaca o cordones de algodón, teñidos y tejidos mediante el uso de una compleja secuencia de nudos. Gracias al registro de crónicas coloniales tempranas, es sabido que en épocas incaicas este sistema fue empleado en la recopilación de datos y el mantenimiento de registros, tales como obligaciones tributarias de cada zona, censos de población, información calendárica, producción y organización militar, así como también en la narración de hazañas épicas. No obstante el conocimiento adquirido, el khipu, como sistema de comunicación de carácter mnemotécnico incaico, presenta aún más interrogantes que certezas frente a sus posibles lecturas e interpretaciones. En torno a ello se sabe, gracias a crónicas como la de Felipe Guaman Poma de Ayala (2013 [1615]), que estas cordeletas de colores con distintos tipos de nudos tuvieron dos funciones en el Incario. Una era de carácter contable, en acción conjunta con un tipo de ábaco llamado yupana, cuyas cuentas eran realizadas por un especialista llamado quipucamayoc; y la otra, era servir de soporte a narraciones acerca de hitos de importancia histórica, las que eran "leídas" y transmitidas por dicho especialista. Asimismo, Guaman Poma (2013 [1615]: 828-830) visibiliza la función calendárica y astronómica del quipu, particularmente gracias al dibujo del "indio astrólogo" Juan Yunpa y a la detallada descripción de la actividad astrológica y de registro del tiempo de este.

El origen del quipu, nombre que en quechua significa "nudo", se remonta con toda probabilidad a épocas preincaicas. En efecto, algunos autores sitúan sus antecedentes en culturas tan antiguas como la de Caral (3000-1900 AC), ubicada al norte de Lima, en el valle de Supe, gracias al extenso trabajo liderado por la arqueóloga Ruth Shady y colaboradores (2000), quien encontró un artefacto muy similar a estas cordeletas en un contexto funerario (Ramírez 2015: 120). Para Nolan y Urton (2017: 280), se trataría de un género específico de quipu, denominado "de donación u ofrenda". También se han encontrado evidencias de instrumentos similares en contextos culturales Wari (600-1100 DC) (Lolas E. 2018). Al respecto, existe una discusión concerniente a la estructura de los quipus del Horizonte Medio o Wari y los quipus de otros períodos. Es así que William Conklin considera únicamente los artefactos de este período como una herramienta mnemotécnica; Asher y Asher sostienen un carácter general basado en un sistema de notación, y Grube, Arellano y Urton afirman la existencia de un significante de información sintáctica y semántica contenida en todos los quipus (Pereira-González \& Batallas-Berdón 2019: 72).

Entre los muchos autores que han tratado el tema -además de Gary Urton, quien le ha dedicado más de 
cuarenta años- cabe destacar los aportes de Marco Curatola y José Carlos de la Puente, quienes han discutido acerca de la posible lectura y escritura de quipus a través de distintas operaciones y protocolos que permitirían "contar y registrar al mismo tiempo" (Curatola \& De la Puente 2013: 195). Por lo tanto, no existiría una única forma de escribir y leer estas cordeletas, sino diversas variantes en función de especificidades culturales, locales y temporales. Esta característica hablaría de un proceso dinámico o generativo de formas de registro y lectura, asociable a lo que Frank Solomon llamó "reactivación ritual"; es decir, el hecho de "volver a contar" (Curatola \& De la Puente 2013: 196). Estas ideas son compartidas por Nolan y Urton (2017: 274), quienes cuestionan la existencia de un patrón o una "forma" estandarizada de confección y lectura del quipu. Con respecto a su uso contable, Nolan y Urton (2017: 276) señalan que estas cuerdas contenían valores numéricos y otros tipos de contenidos codificados por nudos, según un sistema posicional con base diez, además de establecer otros códigos de alternancia lingüística, como "marcados" y "no marcados". Cabe mencionar también el trabajo de Carmen Arellano y Gary Urton (2011), en el que analizan más de 304 quipus o sus fragmentos, pertenecientes a la colección custodiada en el Museo Nacional de Arqueología, Antropología e Historia de Perú.

La particularidad del quipu andino, en cuanto a su estructura, reside en que cada nudo atado en la parte inferior del colgante o tramo principal indica una unidad, en tanto los nudos atados en grupos hacia arriba, señalan, respectivamente, decenas, centenas y millares. Según Nolan y Urton (2017), el quipu emplea el sistema decimal, aun cuando están ausentes algunos símbolos explícitos equivalentes, como el cero del sistema numérico arábico. Estos cordeles, además de la particularidad de sus nudos, poseen otros atributos, como la diversidad de colores y las alternativas de torsión en sentido de una "S" o de una "Z" (Nolan \& Urton 2017: 281).

\section{SOBRE EL ORIGEN Y ANTIGÜEDAD DEL QUIPU MAPUCHE O PRON}

El quipu, al menos en su función contable, ya estaba presente en Chile central al momento de la llegada de los españoles, como lo evidencia Jerónimo de Vivar (1966 [1558]: 51) al referir sucesos previos al asalto indígena de Santiago del 11 de septiembre 1641. Vivar señala que, durante el trayecto que emprende Pedro de Valdivia con su hueste al valle de Aconcagua, a cuatro leguas de la ciudad, en el valle de Colina, las fuerzas españolas capturaron a dos indios vigías que observaban la columna militar desde una peña. Al interrogarlos, estos "luego confesaron su intención y mostraron un quipo, que es hilo grueso con sus nudos, en el cual tenían tantos nudos hechos cuantos españoles habían pasado" (Vivar 1966 [1558]: 51).

No debe sorprendernos la presencia de este instrumento de registro en los valles centrales chilenos antes de la irrupción europea, pues existe consenso de que se trata de un área que se encontraba bajo el dominio político inca, a lo menos y según los más conservadores, hasta el río Maipo. El propio Vivar (1966 [1558]: 39) nos dice que Quilicanta -quien habría dado la orden de contabilizar a quienes componían la columna española con ayuda de un quipu- era un gobernador "puesto por el Inca en esa tierra”. Por otra parte, ya en el último decenio del siglo xvi, García Óñez de Loyola (1960 [1593]: 259-267), luego de asumir el mando de la gobernación de Chile, instruye a los administradores de los bienes de los indios para que en cada pueblo el cacique "que más razón tuviere" poseyera un "quipo" en el que registrara los movimientos de los bienes de su comunidad.

Sin embargo, los otros cronistas chilenos del siglo XVI no hacen mención del quipu. En efecto, las siguientes referencias sobre este artefacto aparecen con el cambio de siglo y provienen del Maestre de Campo González de Nájera (1889 [1614]: 98), quien permaneció en Chile cumpliendo funciones militares en la frontera del Biobío entre 1601 y 1607.

La descripción de González de Nájera da cuenta de un instrumento de cómputo de tiempo para acciones guerreras; es decir, se trata un artefacto calendárico utilizado en un contexto militar fronterizo por los mapuches del sur: ${ }^{3}$

Lleva el mensajero una cuerda a que llaman yipo, de tantos nudos cuantos días han de tardar los indios en venir a juntarse en el puesto que se les declara, para lo cual van deshaciendo cada día un nudo, contando los que faltan para conforme ellos medir el tiempo de sus jornadas y ajustar el en que han de llegar a lugar señalado. Pasa de cacique en cacique por todas las provincias, como si volara la anunciadora insignia de guerra que lleva el mensajero, que es una ensangrentada saeta, y para tocar el arma más viva, la suele acompañar cabeza de español, si acaso les ha caído alguno en las manos (González de Nájera 1889 [1614]: 98). 
Nótese la similitud en la función -contable en los tres casos- y en la descripción del artefacto: "hilo grueso" (Vivar) o "cuerda" (González de Nájera); por tanto, los nudos representan unidades de registro, sean estas "hombres", "bienes" (Vivar y García Óñez de Loyola) o "días" (González de Nájera).

La cualidad de artefacto -asociado simbólica y funcionalmente a la guerra- que nos detalla González de Nájera, será una característica predominante en las referencias etnohistóricas posteriores del quipu mapuche, que llegan hasta fines del siglo xIx y principios del xx: Núñez de Pineda y Bascuñán (1863 [1673]), Rosales (1877 [1674]), Pietas (1846 [1729]), Stevenson (1825) y Moesbach (1930). Aunque otros autores refieren también su uso más genérico como registro contable, calendárico o mnemotécnico en un contexto civil. Así, por ejemplo, al hablar de la "calidad" de los habitantes de Chile, Ovalle expresa:

Estos quipos son sus libros de memoria, y quentas, y con estos, las dan de mucha cantidad de ganados, con distinción de los que se han muerto de enfermedad, o de otros qualquiera accidentes, de los que se han dado, o consumido en el sustento de la casa, y de los pastores; con estos dan razón de los sucedido en tal, y tal ocassión, y tiempo, y de lo que hizieron, hablaron, y pensaron; y quando se confiessan, les sirven de apuntamiento para mexor acordarse de sus pecados, y dezirlos con toda distinción, y claridad; ayúdales para esto mucho la felicidad de su memoria, que la tienen tan retentiva [...] (Ovalle 1646: 92-93).

Sospechamos que, en la escena que nos presenta Ovalle, se trata de mapuches que viven en tierra de españoles ("indios cristianos o reducidos") a mediados del siglo XVII, posiblemente en Chile central. Esto en razón del tipo de actividades que describe (labores domésticas, ritos católicos), pues él tuvo poco contacto con los mapuches del sur. Sin embargo, a principios del siglo siguiente, entre 1712 y 1714, el viajero Frezier (1902 [1716]: 42) describe una función en parte similar en un área bastante fronteriza, como lo era Talcahuano por esas fechas, diciendo sobre este instrumento que lo usan "para llevar la cuenta de su ganado i conservar el recuerdo de sus asuntos particulares". Por último, en su Calepino hispano-chileno, el jesuita Febrés señala el uso calendárico del pron por los mapuches del sur a principios de la segunda mitad del siglo xviıI más allá del contexto militar: "los ñudos, que hacen en un hilado para contar los días, que faltan para alguna junta, ò bèbida, ò juego, ò también por las pagas de una muerte, ò hurto, y si van con hilado colorado, es decir, que à sangre, y à fuego han de dar las pagas" (Febrés 1765: 602).

\section{ADICIONANDO EL DATO ARQUEOLÓGICO}

En el interesante trabajo de Blanco, Peñaloza y De la Maza (2015) sobre el arte rupestre del centro-sur de Chile, en parte coetáneo a la presencia inca, los autores destacan la evidente similitud de diseños entre algunos petroglifos del denominado estilo Guaiquivilo, en espacios cordilleranos andinos de Chile central, principalmente entre las cuencas de los ríos Cachapoal y Maule (Blanco et al. 2015: 102), y los quipus (fig. 1). Sin datación cronológica absoluta, Niemeyer y Weisner propusieron situar estos petroglifos en un rango comprendido entre los siglos XII a XVI (Blanco et al. 2015: 96).

En este mismo estudio, Blanco, Peñaloza y De la Maza recuerdan que esta asociación entre arte rupestre cordillerano y quipu ya había sido señalada para el cajón de Los Cipreses, afluente del río Cachapoal, en un trabajo pionero de Jaffuel (1930).

Al revisar el trabajo del padre Jaffuel, encontramos algunas ideas que nos parece interesante exponer. Cabe destacar que el escrito de Félix Jaffuel reporta una expedición al cajón de Los Cipreses del año 1908, es decir, 22 años antes de su publicación, y reproduce una carta del padre Calmes, quien también participó de la expedición y, en principio, debía publicar al respecto. En esa carta dice Calmes:

\footnotetext{
Provisoriamente, me atengo a la hipótesis que emití desde el principio: el paraje donde existen las inscripciones ha sido asiento de un lavadero de oro. Los mineros no podían explotarlo sino en una época del año, es decir, en la época en que se derriten las nieves y las aguas abundantes. Dada esta circunstancia, cada minero o grupo de mineros se habrá visto obligado a señalar el sitio preciso cuya explotación le correspondía a fin de reconocerlo fácilmente a cada vuelta de estación y evitar así posibles conflictos. Con este fin se habrá dividido la región aurífera en placers [placeres] como diríamos ahora, dando a cada uno su marca propia grabada en la piedra y probablemente también señalando en ella la extensión de cada concesión (Jaffuel 1930: 242).
}

La hipótesis de Calmes es muy sugerente y plantea una función de tipo cartográfica para los petroglifos del valle de Los Cipreses, cuyas inscripciones estarían relacionadas unas con otras e incluirían una mayor, la "Piedra 



Figura 1. Posibles quipus y nudos Guaiquivilo (Blanco et al. 2015: 102). Figure 1. Possible Guaiquivilo quipus and knots (Blanco et al. 2015: 102).

del Indio", que supone Calmes, podría ser interpretada como "plano general" (Jaffuel 1930: 242-243).

No sabemos si la afirmación de Calmes de que el valle de Los Cipreses fue asiento de lavaderos de oro se basa en alguna evidencia encontrada, o en el conocimiento de actividad aurífera en la zona, o si lo plantea simplemente como parte de su hipótesis sin un respaldo empírico. Hasta donde hemos averiguado, no hay registro oficial de placeres auríferos allí, aunque sí se encuentran en el área yacimientos de cobre. ${ }^{4}$
Aunque el padre Calmes plantea que estos petroglifos parecieran ser del Período Hispánico -basado en la inferencia del uso de un cincel metálico en su elaboración- hoy sabemos que, instrumentos de metal de este tipo fueron usados en el Período Prehispánico Tardío en toda esta área (Uribe \& Sánchez 2016: 562). Por lo demás, Calmes reconoce la factura andina de estas inscripciones (Jaffuel 1930: 244).

Terminada la reproducción de la carta de Calmes, Jaffuel agrega que los conjuntos de líneas paralelas di- 


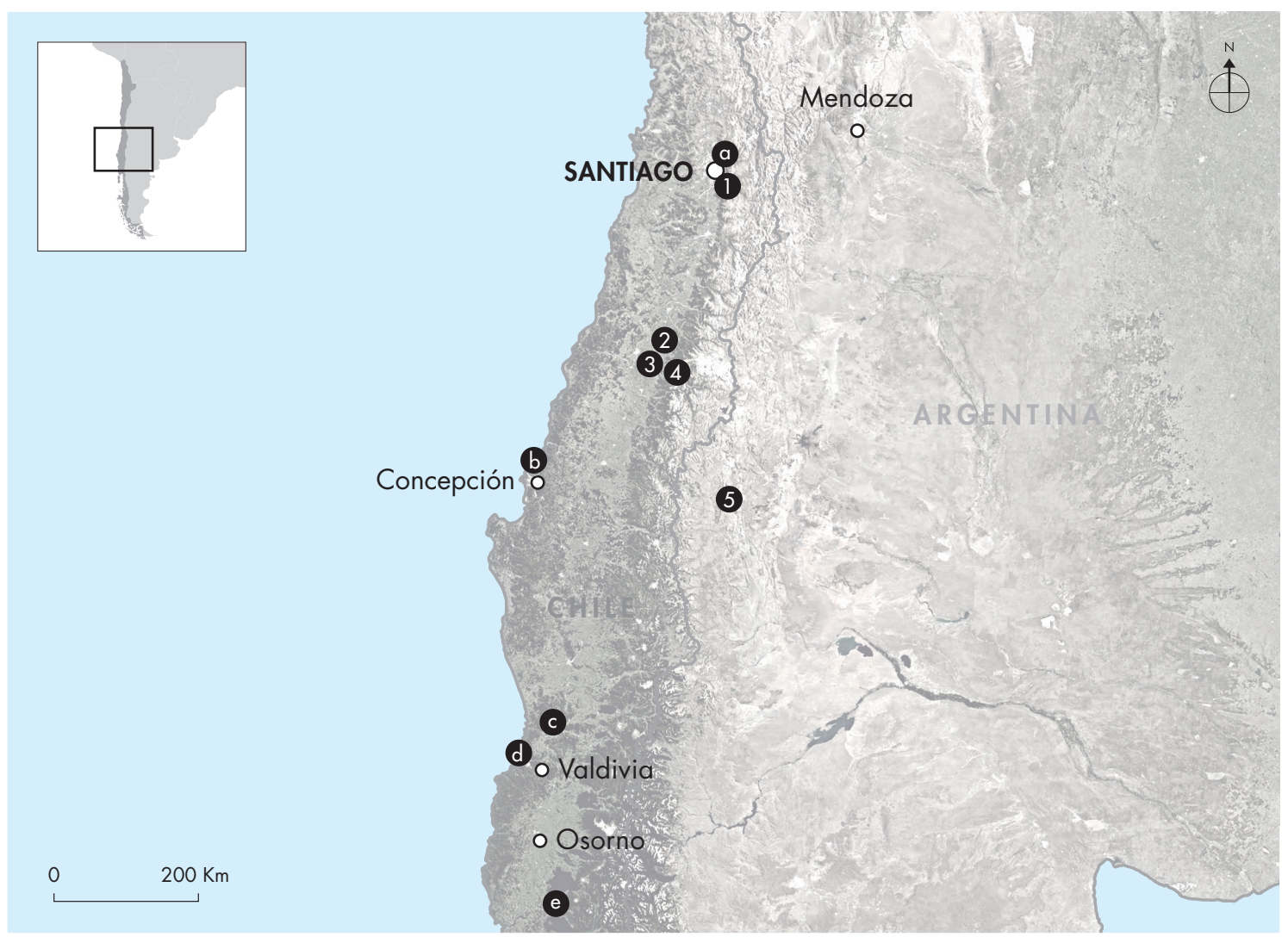

Figura 2. Lugares asociados a quipus y sitios con petroglifos estilo Guaiquivilo: Quipus: a) Colina (1558); b) Talcahuano (1714); c) Mariquina (1656); d) Valdivia (1792); e) Llanquihue (1778). Petroglifos: 1) Cajón Los Cipreses; 2) Cajón Calabozos; 3) Cerro Quiñe; 4) La Gloria; 5) Colo Michi có. (Elaboración: Francisco Javier Medianero). Figure 2. Places associated to quipus and sites with Guaiquivilo style petroglyphs Quipus: a) Colina (1558); b) Talcahuano (1714); c) Mariquina (1656); d) Valdivia (1792); e) Llanquihue (1778). Petroglyphs: 1) Cajón Los Cipreses; 2) Cajón Calabozos; 3) Cerro Quiñe; 4) La Gloria; 5) Colo Michi có. (Map elaborated by Francisco Javier Medianero).

bujados en las piedras de Los Cipreses se asemejarían a los quipus incásicos, de allí la idea expresada por su correligionario de que quienes los fabricaron y entendieron debieron ser "indios venidos del Perú" (Jaffuel 1930: 244).

En resumen, la hipótesis de Calmes, compartida por Jaffuel, supone que los petroglifos de Los Cipreses cumplen una función cartográfica asociada a lavaderos de oro, que esta cartografía contiene, en parte, simbología -y por lo tanto información- característica de los quipus, y que quienes hicieron dichos diseños, así como quienes los interpretaron, conocían los códigos inscritos en los quipus.

Respecto de la relación entre quipu y cartografía, conviene agregar que algunos autores ya han señalado esta cuestión para el mundo andino. Por ejemplo, Porfirio Miranda (1958: 118, 121), además de precisar que los quipus indicaban la ubicación de lugares de riquezas acumuladas, refiere la existencia de un tipo de quipu especial para señalar ubicación de caminos y poblaciones. Por otra parte, Margot Beyersdorff (2002: 32), en su análisis de cartografías andinas -papelones- del último decenio del siglo xvi en la provincia de Yauyos, departamento de Lima, afirma que estas reproducen información topográfica contenida en los quipus.

Es interesante también lo que se dice en el manuscrito de Huarochirí sobre la función de los quipus en el registro del oro y la plata que se ofrendaba a las waka:

Hinataqsîi ingakunari tukuy hinantin wakakunaman alli riqsiŝqa wakamanqa qurinta qullqinta kipullamanta tukuy hinantin wakakta quchiq karqan". (De la misma manera, los ingas mandaban ofrendar, conforme a sus quipus, su oro y su plata a todos los huacas, a todos los huacas de gran prestigio, a todo el conjunto de los huacas) (Anónimo 1613?). 
Por nuestra parte, creemos que la hipótesis de CalmesJaffuel es meritoria y una pista de investigación interesante de seguir. ${ }^{5}$ Esto considerando que el estilo Guaiquivilo -al que se han asociado los petroglifos de Los Cipresesabarca a lo menos hasta los valles andinos occidentales del extremo sur de la cuenca del Maule, y que también se ha vinculado a valles andinos argentinos de más al sur, en particular a sitios del departamento de Minas, los petroglifos de Colo Michi-có (Vega et al. 1996), a la altura de Chillán-Laja del lado chileno, en un sector de actividad aurífera intensa (fig. 2).

En definitiva, y volviendo al tema principal, si aceptamos la idea de que ciertos diseños de petroglifos cordilleranos de Chile central reproducen quipus y que su datación es prehispánica, el registro arqueológico estaría apoyando la información etnohistórica de una presencia y difusión amplia del quipu en Chile, a lo menos hasta el sector sur del río Maule.

La cuestión que queda pendiente es saber si la presencia del quipu en Chile central y sur se debe a los incas o existía con anterioridad. Arellano (2009: 230), quien asume en principio que la procedencia del quipu mapuche es andina y habría llegado con los incas, deja abierta la posibilidad de un ingreso más temprano, en épocas del Horizonte Medio o en tiempos de los wari/ tiwanaku, hacia el 700-1100 DC. Esta idea de un origen andino preincaico del quipu chileno coincide con lo expresado ya hace muchos años por Max Uhle (1912: 268) y Martín Gusinde (1916: 87-88) respecto de la existencia de un influjo cultural andino muy antiguo entre los mapuches. Trabajos recientes han aportado más antecedentes con relación a una profundidad histórica preinca de características culturales mapuches que coinciden con tradiciones andinas, ya sea en el ámbito de lo lingüístico-ideacional (Moulian et al. 2018) como en lo relativo a la complejidad agrícola (Dillehay et al. 2007).

\section{A PROPÓSITO DEL NOMBRE MAPUCHE PARA EL QUIPU EN ESTE DEBATE}

La designación mapuche para el quipu (pron) al igual que su equivalente en lengua quechua, significa nudo. La escritura del término presenta diversas variantes ortográficas, en particular a partir de los trabajos lingüísticos de Lenz y de los misioneros alemanes de principios del siglo $\mathrm{xx}$ que trataron de fijar de mejor manera la fonética y particularidades dialectales del mapudungun, dando paso así a variantes escriturales tales como: PORON (Lenz 1895-1897: 28, Augusta 1910: 27), PËRON (Moesbach 1930: 271) y PÜRON (Arellano 2009: 231). ${ }^{6}$

Es decir, el mapudungun prefirió su propio léxico para designar un artefacto que habría tenido un origen andino. ${ }^{7}$ Si se parte del supuesto que designar un objeto cultural de origen foráneo con su propio vocabulario supone un arraigo profundo $y$, por lo tanto, una incorporación muy antigua de dicho artefacto, podríamos plantear la hipótesis de que el quipu aparece entre los mapuches incluso antes de la irrupción inca.

Un hecho que contradice esta hipótesis es que, en las tres referencias más tempranas (Vivar 1966 [1558], García Óñez de Loyola (1960 [1593]), González de Nájera 1889 [1614]), el artefacto es mencionado con su designación quechua y no mapudungun. Se podría argüir que esto ocurre porque los tres cronistas simplemente desconocían el término nativo o bien porque, conociéndolo, prefirieron el vocablo más difundido. Sin embargo, lo extraño es que alguien con tanto conocimiento de la lengua y protocolos mapuches como el padre Luis de Valdivia no entregue en su vocabulario bilingüe de 1606 el significado de quipu para la voz "pron", que registra, efectivamente, como equivalente de "nudo" (Valdivia 1606: s.p.).

También podría ser que, existiendo ya una versión del quipu en Chile antes de la llegada de los incas, estos hayan introducido una nueva, quizás más sofisticada, razón por la cual habría prevalecido en Chile central su designación en la lengua del incario: quipu; y que la versión más antigua del artefacto se haya conservado entre los mapuches del sur bajo la designación nativa, pron, no reportada hasta mediados del siglo xvir. En efecto, será el jesuita Diego de Rosales quien nos informará, ya avanzado el siglo xviI, del término mapuche para quipu al describir las tradiciones militares de los habitantes de la Araucanía. En concreto, Rosales se refiere al quipu que se utiliza para convocar a la guerra y por ello se trata de un "cum-pron", es decir de un quipu "rojo" que se hace circular junto con la denominada "flecha ensangrentada", indicador indudable de alzamiento (Rosales 1877: 122). ${ }^{8}$

El término pron será usado corrientemente por los autores del siglo xviII para referirse a la cordeleta mapuche; por ejemplo, en Pietas (1846 [1729]), Febres (1765) y Delgado9 (1846 [1778]). El significado del tér- 
mino en cuanto aparato mnemotécnico está presente en la memoria oral mapuche hasta la actualidad, aunque todo indica que se ha perdido su uso, como lo señala Arellano (2009: 237).

\section{SOBRE EL USO DEL PRON}

Queda claro hasta ahora que el pron era un instrumento de cómputo contable, calendárico y mnemotécnico. Arellano (2009: 233-234) piensa que, posiblemente, pudo haber tenido una función escritural no registrada por las fuentes coloniales y posteriores, debido al sesgo ideológico de estas, cuestión que no podemos responder en el estado actual de los datos.

Hemos analizado doce referencias coloniales sobre el pron, de 1558 a 1792, de ellas, nueve refieren su uso calendárico, cinco una función contable y dos como útil de apoyo mnemotécnico narrativo (tabla 1). ${ }^{10}$ Por otra parte, respecto del ámbito de uso, se puede apreciar en la misma tabla que en nueve de las doce referencias el pron está asociado a un contexto militar, en dos a un contexto civil, en uno también se incluye el ámbito religioso católico y en dos hay referencias a aspectos de conservación de la memoria histórica.

Finalmente, en cuanto a los usuarios del pron -cuando estos se especifican de alguna manera (en diez casos) - en ocho se hace referencia explícita a denominaciones o funciones indígenas relativas a la guerra, en dos se entiende que quienes manejan el pron son "expertos" o autoridades indígenas (1593 y 1714) $y$, en un último caso, quien aparece usando un pron es un intérprete-mediador y baqueano no indígena, un Capitán de Amigos (1778). Todo lo anterior se puede apreciar en la tabla 1 , donde puede observarse también la localización geográfica para cada referencia.

\section{SOBRE LA MATERIALIDAD Y FUNCIONAMIENTO DEL PRON}

La información colonial recopilada registra la existencia de pron de estructura simple (un hilo) y de composición compleja (varios hilos). Para el caso de pron simples de un hilo, hemos encontrado seis referencias explícitas que cubren todo el período colonial. En cuanto a los pron de varios hilos, se explicitan en cuatro casos: uno para el siglo XVI y tres para el siglo XVIII (tabla 2). De los cuatro registros de pron complejos, en dos de ellos se señala la existencia de un soporte desde el cual penden otros hilos; este soporte es también un hilo para el caso de 1729-1; en cambio, para la descripción de 1792, es un trozo de madera.

Respecto del uso de colores, en seis ocasiones se señala la presencia de más de un color, ya sea porque los pron simples pueden ser de diferentes colores (1656) o bien porque los pron complejos están compuestos de hilos de colores (siglo XVIII). En dos casos de uso de colores, no es posible saber si la variabilidad cromática hace alusión a un pron simple o complejo (1729-2 y 1765). Cuando se especifica la cantidad de colores usados, esta es siempre equivalente a cuatro y el color que más se repite es el rojo, asociado indiscutiblemente al uso de la violencia (tabla 2).

Sobre la función de los colores, las referencias dan cuenta de que la diferenciación cromática es una técnica que permite discriminar entre tipos de acontecimientos u objetos registrados en los hilos. De este modo, en los registros de 1729 ( 1 y 2) se describe una relación entre colores y macro-alianzas político-territoriales de la Araucanía, los butanmapu, donde correspondería un color a cada una de estas cuatro macro-alianzas. Por otra parte, para el caso de 1792 -que analizaremos en detalle en el apartado siguiente- los colores dan dos tipos de información: calendárica (día cero: nudo negro; día final: nudo blanco) y política (aprueban: nudos rojos; rechazan: nudos rojos con azul) (tabla 2).

El tipo de cómputo es otra información que podemos obtener de estas referencias. La cuenta se lleva de dos maneras: a) aditiva, en cuyo caso las unidades de cómputo que son los nudos se van adicionando para aumentar una cuenta; y b) sustractiva, en la que los nudos se van deshaciendo a medida que transcurren unidades de tiempo en función de un plazo o fecha tope.

Claramente, el modo aditivo se da en casos de contabilidad de personas o bienes: registros de 1558 y 1729-2. En cambio, el cómputo sustractivo es característico de un útil calendárico en función de una fecha futura: ocurrencias de 1600, 1629, 1656 y 1729-1. El pron usado en 1792 parece excepcional, pues contendría las dos modalidades: dos hilos tienen una función contable y los otros dos, calendárica. 
Tabla 1. Uso, usuarios y contexto de uso del pron, 1558-1792. (Elaboración: José Manuel Zavala). Table 1. Uses, users and context of use of the pron, 1558-1792. (Table elaborated by José Manuel Zavala).

\begin{tabular}{|c|c|c|c|c|c|}
\hline $\begin{array}{c}\text { FECHA DEL } \\
\text { REGISTRO Y ÁREA } \\
\text { GEOGRÁFICA }\end{array}$ & $\begin{array}{l}\text { DESIGNACIÓN } \\
\text { DEL ARTEFACTO }\end{array}$ & USO & USUARIOS & CONTEXTO & FUENTE \\
\hline $\begin{array}{c}1558 \\
\text { Colina }\end{array}$ & Quipo & Contable & Vigías & Militar & $\begin{array}{c}\text { Vivar } \\
1966 \text { [1558]: } 51\end{array}$ \\
\hline $\begin{array}{l}1593 \\
\text { Chile }\end{array}$ & Quipo & Contable & $\begin{array}{c}\text { Cacique / } \\
\text { Cuadrillero de minas }\end{array}$ & Civil-económico & $\begin{array}{c}\text { García Óñez de Loyola } \\
1960 \text { [1593]: 261-267 }\end{array}$ \\
\hline $\begin{array}{c}1607 \\
\text { Araucanía }\end{array}$ & Yipo & Calendárico & Mensajero & Militar & $\begin{array}{c}\text { González de Nájera } \\
1889 \text { [1614]: } 98\end{array}$ \\
\hline $\begin{array}{c}1629 \\
\text { Araucanía }\end{array}$ & Nudos & Calendárico & Mensajero & Militar & $\begin{array}{c}\text { Núñez de Pineda \& B. } \\
1863[1673]\end{array}$ \\
\hline $\begin{array}{l}1646 \\
\text { Chile }\end{array}$ & Quipo & $\begin{array}{c}\text { Calendárico, } \\
\text { contable y } \\
\text { mnemotécnico }\end{array}$ & No especifica & $\begin{array}{c}\text { Civil, religioso } \\
\text { católico, memoria } \\
\text { histórica }\end{array}$ & $\begin{array}{c}\text { Ovalle } \\
\text { 1646: } 92-93\end{array}$ \\
\hline $\begin{array}{c}\text { 1650 } \\
\text { Araucanía }\end{array}$ & Cumpron & Calendárico & $\begin{array}{l}\text { Toqui General / Leb- } \\
\text { Toqui (su mensajero) }\end{array}$ & Militar & $\begin{array}{c}\text { Rosales } \\
1877[1674]: 112-113\end{array}$ \\
\hline $\begin{array}{c}1656 \\
\text { Mariquina }\end{array}$ & Pron & Calendárico & Cacique / Toqui & Militar & $\begin{array}{l}\text { Acuña \& Cabrera, citado } \\
\text { en Obregón 2016: } 113\end{array}$ \\
\hline $\begin{array}{l}1714 \\
\text { Talcahuano }\end{array}$ & Quipo & Mnemotécnico & Expertos & $\begin{array}{l}\text { Memoria } \\
\text { histórica }\end{array}$ & Frezier 1902 [1716]: 42 \\
\hline $\begin{array}{c}\text { 1729-1 } \\
\text { Araucanía }\end{array}$ & Pron & Calendárico & $\begin{array}{c}\text { Toqui General / } \\
\text { Cones (mensajero } \\
\text { x butanmapu) / } \\
\text { Caciques }\end{array}$ & $\begin{array}{c}\text { Militar } \\
\text { (levantamiento } \\
\text { general) }\end{array}$ & $\begin{array}{c}\text { Pietas } 1846 \\
\text { [1729]: } 490-492\end{array}$ \\
\hline $\begin{array}{c}\text { 1729-2 } \\
\text { Araucanía }\end{array}$ & Pron & Contable & $\begin{array}{c}\text { Caciques que } \\
\text { aprueban la guerra }\end{array}$ & $\begin{array}{c}\text { Militar } \\
\text { (levantamiento } \\
\text { general) }\end{array}$ & $\begin{array}{c}\text { Pietas } 1846 \\
\text { [1729]: 490-492 }\end{array}$ \\
\hline $\begin{array}{c}1765 \\
\text { Araucanía }\end{array}$ & Pron & Calendárico & No especifica & Civil y militar & Febres 1765: 602 \\
\hline Llanquihue & Pron & $\begin{array}{l}\text { Calendárico } \\
\text { y contable }\end{array}$ & $\begin{array}{l}\text { Capitán de } \\
\text { Amigos }\end{array}$ & Civil & $\begin{array}{c}\text { Delgado } 1846 \\
{[1778]: 451,466-467}\end{array}$ \\
\hline $\begin{array}{c}1792 \\
\text { Valdivia }\end{array}$ & Quipu & $\begin{array}{l}\text { Calendárico } \\
\text { y contable }\end{array}$ & Mensajero & Militar & Stevenson 1825: 50-51 \\
\hline
\end{tabular}




\begin{tabular}{|c|c|c|c|c|c|c|c|c|c|c|c|c|c|}
\hline 至 & 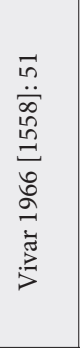 & 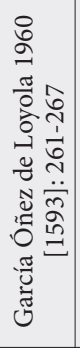 & 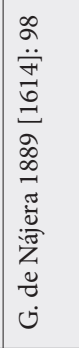 & 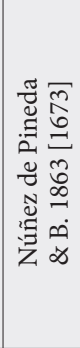 &  & 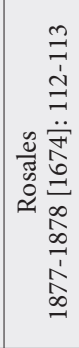 & 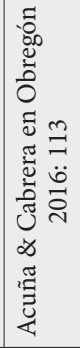 & 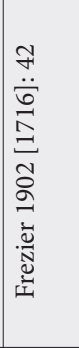 &  &  & 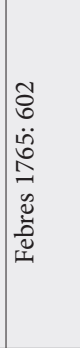 &  & 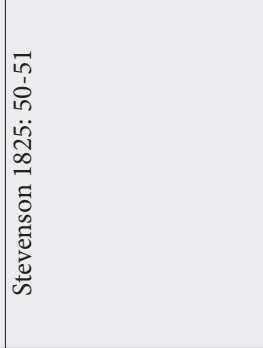 \\
\hline 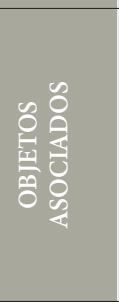 & 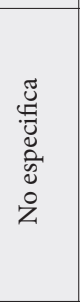 & 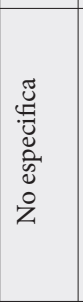 & 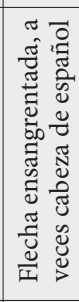 & $\begin{array}{l}\frac{\pi}{\tilde{U}} \\
\frac{\tilde{U}}{I}\end{array}$ & 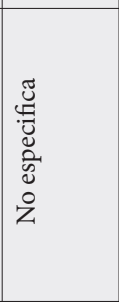 & 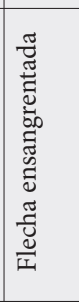 & 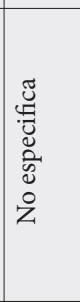 & 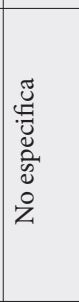 & 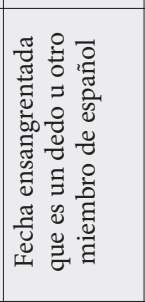 & 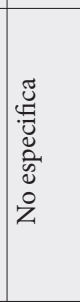 & 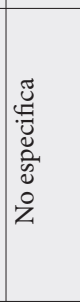 & 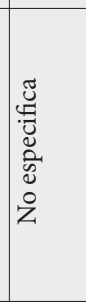 & 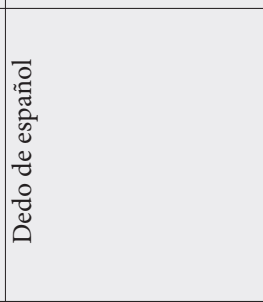 \\
\hline  & 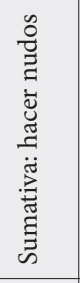 & 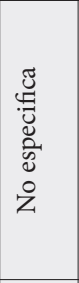 &  & 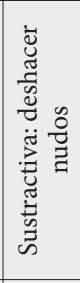 & 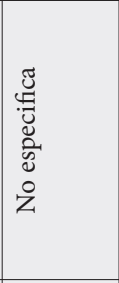 & 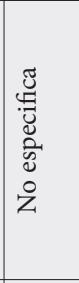 & 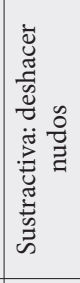 & 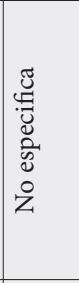 & 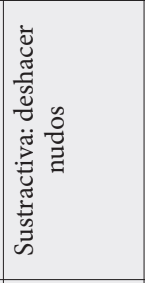 & 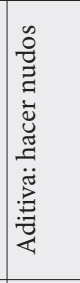 & 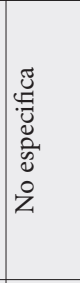 & 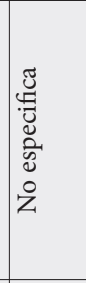 & 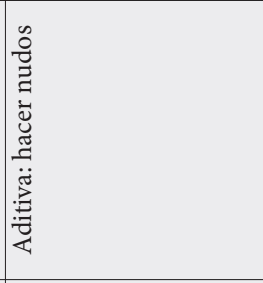 \\
\hline 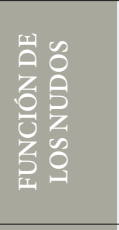 &  & 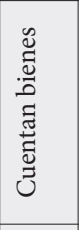 & 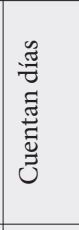 & 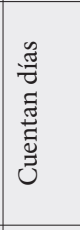 & 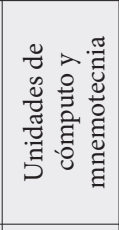 & 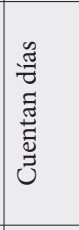 & 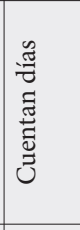 & 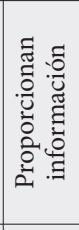 & 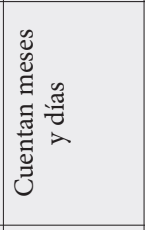 & 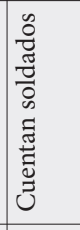 & 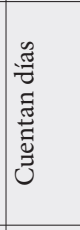 & 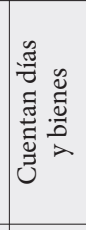 & 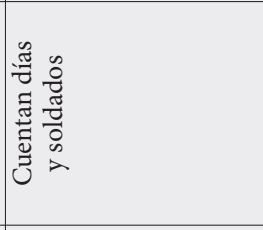 \\
\hline 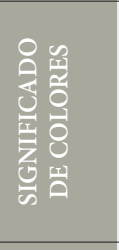 & 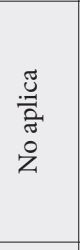 & 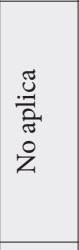 & 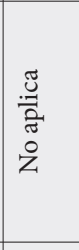 & 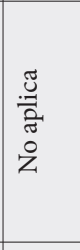 & 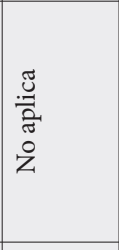 & 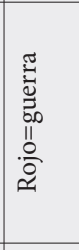 &  & 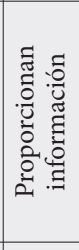 & 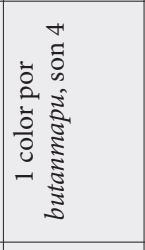 & 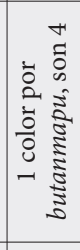 &  & 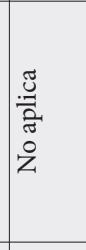 & 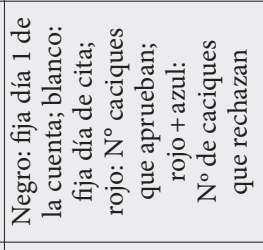 \\
\hline 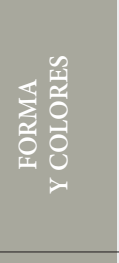 & 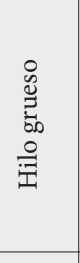 & 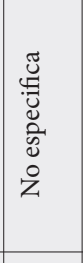 & 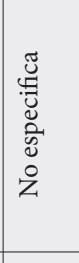 & 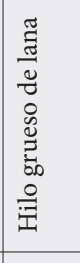 & 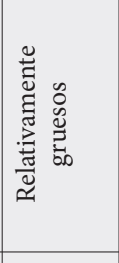 & 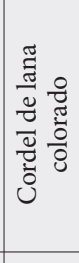 & 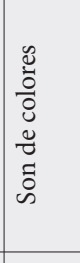 & 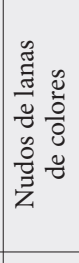 & 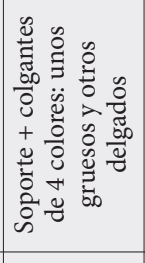 & 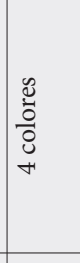 & 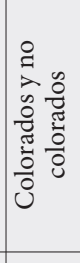 & 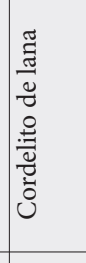 & 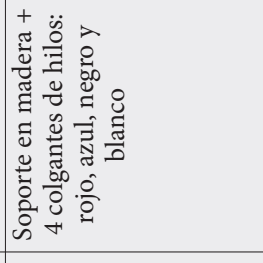 \\
\hline 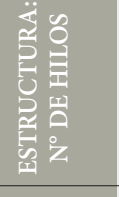 & - & 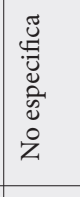 & - & -7 & $\vec{\wedge}$ & - & - & $\vec{\wedge}$ & $\overrightarrow{\hat{A}}$ &  & 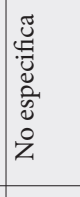 & - & $\begin{array}{l}+ \\
\pm \\
+\end{array}$ \\
\hline 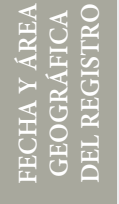 & 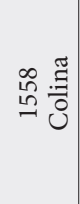 & 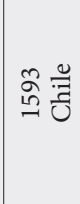 & 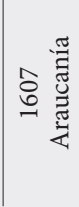 & 突 & 늉 : & 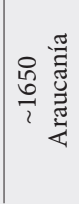 & 营 &  & 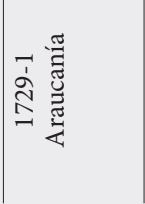 & 证 & 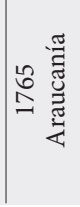 & 离 &  \\
\hline
\end{tabular}




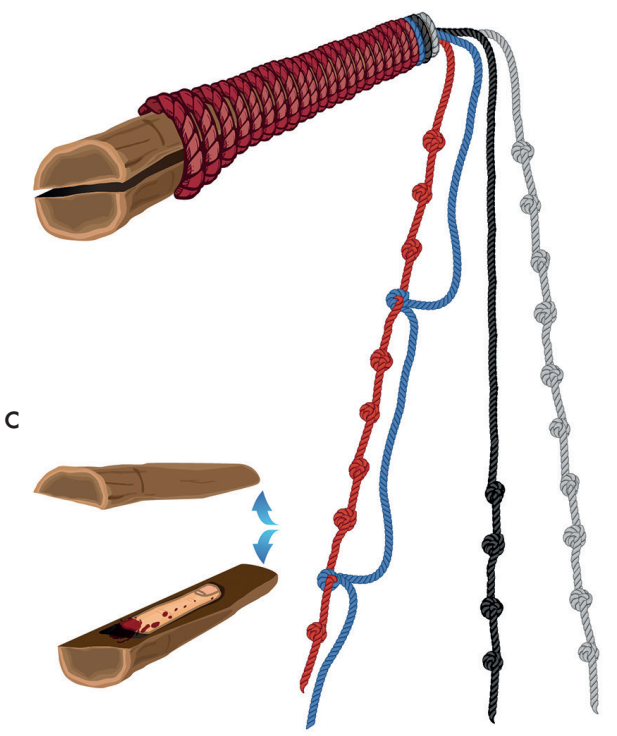

b

\section{d}

e

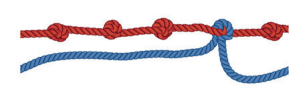

$f$

g

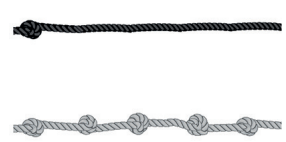

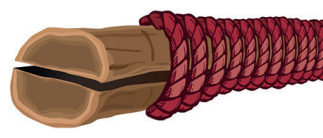

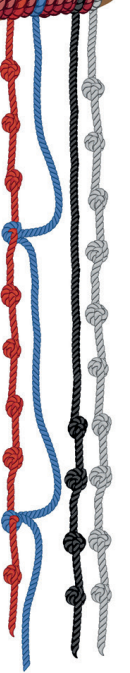

Figura 3. Recreación del pron de Lepitrarn de 1792 según la descripción de Stevenson (1825): a) versión A del pron: “de cola”; b) versión B del pron; "perpendicular"; c) detalle del soporte de madera con el dedo de un enemigo ( ¿flecha ensangrentada"?); d) nudos en hilo rojo cuentan participantes; e) nudos de hilo rojo con azul cuentan no participantes; f) nudos en hilo negro establecen la fecha de partida de la convocatoria; g) nudos en hilo blanco establecen fecha de la convocatoria. (Diseño: Daniel Videla. Concepto: José Manuel Zavala). Figure 3. Recreation of Lepitrarn's quipu from 1792 according to Stevenson's (1825) description: a) Version A of the pron: "from the back";

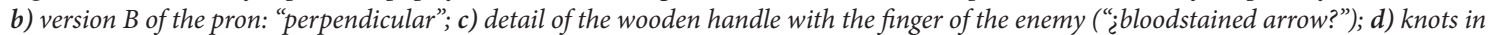
red cord count participants; e) knots in the red and blue cords joined together count non-participants, $f$ ) knots in black cord indicate the date on which the call was sent; $g$ ) knots in white cord indicate the date of the call. (Design: Daniel Videla; Concept: José Manuel Zavala).

\section{EL PRON DE LEPITRARN EN 1792, SEGÚN STEVENSON}

Como ya señalamos, la referencia al uso de un pron en 1792 es muy interesante, pues hasta ahora parece ser la más completa descripción de un quipu mapuche complejo. ${ }^{11}$ El caso es presentado por el viajero Stevenson (1825: 50-51) en su libro sobre los veinte años de residencia en Sudamérica. El autor señala que la descripción de este pron proviene de un juicio realizado en 1792 en la ciudad de Valdivia contra indígenas que habrían estado implicados en la preparación de una sublevación. ${ }^{12}$ Stevenson afirma que el expediente judicial se encontraría en su posesión y lo habría obtenido durante su estadía en dicha ciudad.

José Toribio Medina (1882: 408) encontró suficientemente interesante el caso expuesto por Stevenson como para incluir una traducción en Los aborijenes de Chile. Hemos revisado la traducción de Medina cotejándola con su original en inglés, y notamos que algunas palabras no fueron traducidas o pueden ser interpretadas de manera diferente. Por ello, proponemos una nueva traducción:

En 1972 tuvo lugar una sublevación cerca de Valdivia y, en el juicio de varios de los cómplices, Marican, uno de ellos, declaró que la señal enviada por Lepitrarn era un trozo de madera, de aproximadamente un cuarto de yarda de largo, considerablemente grueso; que había sido hendido, y se encontró que contenía el dedo de un español; estaba envuelto en hilo, con un fleco en un extremo hecho de rojo, azul, negro y blanco; que en el negro estaban atados por Lepitrarn, cuatro nudos, para dar a entender que era el cuarto día después de la luna llena cuando el portador salió de Paquipulli [Panguipulli]; que en el blanco había 10 nudos, para indicar que 10 días después de esa fecha tendría lugar la revolución; que en el rojo debía añadirse un nudo por la persona que lo recibía, si asistía a la revuelta; pero si se negaba, debía hacer un nudo uniendo el azul con el rojo; 
para que, de acuerdo con la ruta determinada por Lepitrarn, pudiera descubrir al regreso de su chasqui, o heraldo, cuántos de sus amigos se unirían a él; y si alguno disentía lo sabría por el lugar ocupado por el nudo que ataba los dos hilos [el azul con el rojo] (Stevenson 1825: 50-51). ${ }^{13}$

El pron de Lepitrarn es un artefacto complejo: calendárico (cuenta días) y contable (cuenta participantes), a la vez combina hilos de colores y nudos. Pero, más allá de esto, nos encontramos frente a un instrumento compuesto de, al menos, tres elementos: hilos de colores, madera y un dedo humano. ¿Cómo se puede explicar esto? Este tipo de quipu se inscribe claramente en la tradición de los sistemas de comunicación mapuche relativos a la guerra. En realidad, podríamos pensar que aquí se presentan dos artefactos condensados en uno: la "flecha ensangrentada" y el "pron".

Existen múltiples referencias en la documentación colonial sobre la "flecha ensangrentada" (Obregón 2016: 106-119) que, junto al pron, circulaba en el territorio mapuche siguiendo determinadas trayectorias para convocar a juntas de guerra, como si ambos artefactos fueran "cartas citatorias" (Rosales 1877 [1674]: 178). En la tabla 2 se observan tres casos, aparte de este de 1792, en que se presenta una asociación entre pron y flecha: 1600,1650 y $1729-1$.

Creemos que el concepto de "flecha ensangrentada" es una categoría altamente simbólica que, no necesariamente era una "flecha ensangrentada", sino que podía tomar la forma de un objeto que representara la flecha: un trozo de madera, por ejemplo. Tampoco se debe pensar que la "flecha" estaba efectivamente cubierta de sangre. A nuestro parecer, lo que se quiere transmitir con la idea de "ensangrentamiento" es que el instrumento que circula fue partícipe (simbólicamente hablando) del sacrificio de un prisionero español. Por lo tanto, quien recibe y acepta esta "flecha" se hace parte de ese acto sacrificial y se une a un pacto secreto indisoluble.

La prueba de ese pacto y del carácter guerrero del llamado (lo "ensangrentado" de la flecha) era la presencia de un trozo del cuerpo del sacrificado, por lo general un dedo, cuando efectivamente se había podido capturar algún enemigo. De otro modo, también es posible pensar que la "flecha" podía contener un sustituto del apéndice humano o bien que bastara con que el color rojo estuviera presente de alguna manera. Por ello, pensamos que el pron de Lepitrarn es una forma de concreción de dos artefactos en uno: la "flecha ensangrentada" y el pron. En efecto, en su composición hay un trozo de madera que constituye el soporte del pron y que representaría a la "flecha"; al interior de este se encuentra el dedo de un español, dándole un carácter de "ensangrentado". El conjunto estaba envuelto con un hilo que, con toda seguridad, debió ser de color rojo, y en un extremo nacían los cuatro hilos de colores que informaban fechas y participantes.

\section{CONCLUSIÓN}

No cabe duda de que el quipu existía entre los mapuches antes de la llegada de los españoles. La cuestión que queda por dilucidar es saber si este fue introducido durante la época Inca o en un período anterior. Posiblemente hubo dos versiones de quipu: una antigua, preincaica, que se habría conservado en los territorios del sur bajo una denominación propia (pron), y otra netamente incaica, introducida con la anexión de Chile central al Tawantinsuyo.

Para el territorio mapuche bajo el dominio inca (Chile central), algunos de los antecedentes expuestos en este trabajo nos permiten plantear la hipótesis de una posible asociación de este artefacto con representaciones petroglíficas que pueden estar ligadas a la identificación de rutas y sitios mineros (Llagostera 1976, Blanco et al. 2015).

Con todo, el uso más conocido del quipu mapuche colonial fue el de un instrumento calendárico y contable que formaba parte de un sistema de organización militar que permitía establecer fechas de encuentro y ataque entre amplios conglomerados, como también determinar número de aliados y combatientes de ambos campos. Por otra parte, no se descarta su uso contable en el ámbito económico y social civil, ni una función mnemotécnica asociada a relatos históricos o míticos, aunque en estos casos las referencias son menos específicas.

En cuanto a su materialidad, los datos nos permiten diferenciar dos tipos de pron, uno simple de un hilo y otro complejo de varios hilos y colores. El pron de Lepitrarn -descrito por Stevenson y recreado en la figura 3- es excepcional, pues muestra una alta complejidad al combinar en un solo instrumento las funciones calendárica y contable, gracias a una asignación de valores diferenciados a colores y nudos. Pero no solo eso, este quipu, además, condensaría dos artefactos en uno: un pron y una "flecha ensangrentada"; es decir, los dos principales componentes simbólicos del sistema comunicativo mapuche asociado a la guerra. 
Agradecimientos Agradecemos a la Agencia Nacional de Investigación Científica y Desarrollo del Gobierno de Chile (ANID), por el financiamiento otorgado a esta investigación en el marco del Proyecto Fondecyt Regular No 1170551 (2017-2020) Tras la ruta del oro. Los habitantes de La Araucanía frente a la ocupación española del siglo XVI, recepción, adaptación y resistencia. Nuestros agradecimientos más sentidos para Alberto Trivero por su amable colaboración a propósito del quipu de Acatanga; a Daniel Videla por su dedicado y profesional dibujo del pron de Lepitrarn; a Tom Dillehay, por su paciente lectura y sabios comentarios, y a Francisco Javier Medianero, por su constante apoyo y colaboración cartográfica. Finalmente, agradecemos sinceramente los comentarios de los evaluadores de este artículo que, sin duda, lo han enriquecido.

\section{NOTAS}

${ }^{1}$ Interdigitación en el sentido de compenetraciones mutuas, sin que necesariamente exista un control político directo (Martínez 1998).

${ }^{2}$ En este trabajo y para el Período Colonial, entendemos por "Araucanía" el territorio mapuche situado entre el río Itata y el lago Llanquihue, con límites variables según las diferentes coyunturas históricas.

${ }^{3}$ Denominamos "mapuches del sur" a los que habitaban del río Itata hacia el sur.

${ }^{4}$ Sernageomin no catastra placeres auríferos en esta zona, pero a unos $42 \mathrm{~km}$ de allí se ubica la mina de cobre El Teniente en un afluente del río Coya, palabra quechua que sirve para denominar una "mina" o "veta mineralógica" (Cobo 1892 [1653], Olivari 1994: 212, Vetter 2007: 45).

${ }^{5}$ La colonización del departamento de Minas de la provincia de Neuquén a fines del siglo XIX está exclusivamente ligada a la extracción aurífera (Belver 2011).

${ }^{6}$ En este trabajo hemos preferido la forma escritural "pron", pues es la variante, sino exclusiva, más usada en las fuentes.

${ }^{7}$ La otra tesis sería que su origen es local, lo que es difícil de sostener.

${ }^{8}$ Cum: colorado, carmesí (Valdivia 1606: s.p.).

${ }^{9}$ Fray Benito Delgado comandó una expedición para descubrir la "ciudad de los Césares" en 1778, partiendo de Valdivia. Fray Delgado exploró la ruta trasandina vía el lago Llanquihue, guiado por el Capitán de Amigos Aburto, quien utilizaba pron para fijar fechas de encuentro con sus interlocutores indígenas.

${ }^{10}$ Las referencias que dan Córdova y Figueroa (1861 [1745]) y Juan Ignacio Molina (1795) acerca del pron, aunque detalladas, son difíciles de circunscribir temporal y geográficamente. Pareciera que más bien se trata de síntesis hechas sobre la base de otros autores. Al cierre de este artículo, hemos localizado otras dos referencias tempranas de quipos: en la Tasa de Santillán (1559) y en un convenio sobre sesmos de indios de Chile central (1566). Se trata de documentos de carácter jurídico que estipulan el uso de quipos por parte de los caciques en las cuentas de sus comunidades; función similar a la señalada por García Ónez de Loyola en 1593.

${ }^{11}$ En efecto, el supuesto quipu "chileno" de Acatanga que se conserva en la colección Michelini (Italia) y que habría sido descubierto en la ciudad de Concepción por el padre Illanes en 1737, es, con toda probabilidad, de factura andina y no chilena. Las circunstancias de su hallazgo son oscuras. Según Laurencich (2016: 83), en 1737 el jesuita Pedro Illanes habría obtenido en la ciudad de Concepción, Chile, de manos del indio Juan Taquic Menéndez de Sodar, el manuscrito que se conoce como Historia y rudimentos de la lengua de los peruanos (1610-1638), junto con el fragmento del quipu de Acatanga. ¿Qué hacía un indígena de nombre más bien quechua en Concepción en época tan tardía y con dicho manuscrito? Es un misterio poco verosímil, por lo que otorgamos escasa credibilidad a esta historia.

${ }^{12}$ Es interesante mencionar que, en el contexto colonial andino, los quipus fueron usados en procedimientos judiciales. Lo informa la crónica de Juan de Matienzo (1967 [1567]) y lo ha estudiado Beyersdorff (2002).

13 "In 1792 a revolution took place near Valdivia, and on the trial of several of the accomplices, Marican, one of them, declared, "that the signal sent by Lepitrarn was a piece of wood, about a quarter of a yard long, and considerably thick; that it had been split, and was found to contain the finger of a Spaniard; that it was wrapped round with thread, having a fringe at one end made of red, blue, black, and white worsted; that on the black were tied by Lepitrarn, four knots, to intimate that it was the fourth day after the full moon when the bearer left Paquipulli; that on the white were ten knots, indicating that ten days after that date the revolution would take place; that on the red was to be tied by the person who received it a knot, if he assisted in the revolt, but if he refused, he was to tie a knot on the blue and red joined together; so that according to the route determined on by Lepitrarn he would be able to discover on the return of his chasqui, or herald, how many of his friends would join him; and if any dissented, he would know who it was, by the place where the knot uniting the two threads was tied" (Stevenson 1825: 50-51).

\section{REFERENCIAS}

ANónimo. 1613? Manuscrito Huarochirí. In Papeles varios sobre los indios incas, huarochiris y otras antigüedades del Perú, C. Molina, vol. 3169, ff. 64v-114v. <http://bdh-rd. bne.es $/$ viewer.vm?id $=0000087346 \&$ page $=1>$ [consultado: 17-03-2021].

Arellano, C. 2009. Un khipu olvidado: el püron mapuche. Notas acerca de su función. In IV Jornadas Internacionales sobre Textiles Precolombinos, V. Solanilla Demestre, ed., pp. 229-240. Barcelona: Universidad Autónoma de Barcelona-L'Hospital de Llobregat. 
Arellano, C. \& Urton, G. (Eds.) 2011. Atando cabos. Lima: Museo Nacional de Arqueología, Antropología e Historia del Perú.

Augusta, F. DE. 1910. Costumbres de los araucanos. Valdivia: Imprenta de la Prefectura Apostólica.

BAHAMONDES, F. 2009. La cerámica prehispánica tardía de Araucanía septentrional: el complejo arqueológico El Vergel y su relación con la hipótesis del proceso de andinización. BA thesis in Archaeology, Departamento de Antropología, Universidad de Chile.

Belver, I. 2011. Cronología del departamento Minas. Neuteca200. <https://sites.google.com/site/neuteca200/ system/app/pages/search?scope=search-site $\& q=$ minas $>$ [consultado: 25-06-2020].

BeyersdorfF, M. 2002. Caminos rituales y cartografía indígena: la vigencia de la Relación de las guacas del Cuzco de Bernabé Cobo en su época. In Incas e indios cristianos: Elites indígenas e identidades cristianas en los Andes coloniales [en línea], J-J. Decoster, ed., pp 39-59. Lima: Institut Français d'Études Andines. <https://doi.org/https://doi. org/10.4000/books.ifea.4076> [consutado: 17-03-2021].

Blanco, J. F., Peñaloza, M. A. \& De la Maza, M. 2015. Memoria inscrita. Arte rupestre de contacto, integración y dominación en el Centro-Sur de Chile. Boletín del Museo Chileno de Arte Precolombino 20 (2): 89-110.

Сово, В. 1892 [1653]. Historia del nuevo mundo. Notas y otras ilustraciones por Marcos Jiménez de la Espada. Sevilla: Impresión de E. Rasco.

Córdova y Figueroa, P. DE. 1861 [1745]. Historia de Chile (1492-1717). Santiago: Imprenta del Ferrocarril.

Cruz, P. 2013. De wak'as, minas y jurisdicciones. Apuntes metodológicos en torno a la territorialidad en tiempos del Inka. In Aportes multidisciplinarios al estudio de los colectivos étnicos Surandinos. Reflexiones sobre QaraqaraCharka tres años después, A.M. Presta, ed., pp. 293-330. La Paz: Plural Editores-Instituto Francés de Estudios Andinos.

Curatola, M. \& De la Puente, J. C. 2013. El quipu colonial. Estudios y materiales. Lima: Fondo Editorial de la Pontificia Universidad Católica del Perú.

Delgado, Fr. B. 1846 [1778]. Diario del R. P. Fr. Benito Delgado, capellán de la espedición que se hizo para el descubrimiento de los Césares. In Documentos sobre la historia, la estadística y la geografía, C. Gay, ed., vol. I, pp. 431-485. Paris: en casa del autor.

Dillehay, T. 2011. Monumentos, imperios y resistencia en los Andes. El sistema de gobierno mapuche y las narrativas rituales. Santiago: Ocho Libros.

Dillehay, T. \& Gordon, A. 1998. La actividad prehispánica de los incas y su influencia en la Araucanía. In La frontera del estado inca, T. Dillehay \& P. Netherly, eds., pp. 183-196. Quito: Fundación Alexander von Humboldt-Editorial Abya-Yala.

Dillehay, T., Pino, M., Bonzani, R., Silva, C., Wallner, J. \& LE Quesne, C. 2007. Cultivated wetlands and emerging complexity in south-central Chile and long distance effects of climate change. Antiquity 81: 949-960.

Febrés, A. 1765. Arte de la lengua general del Reyno de Chile, con un dialogo Chileno-Hispano muy curioso: a que se añade la doctrina christiana, esto es, rezo, catecismo, coplas, confesionario y pláticas; lo más en lengua chilena y castellana: y por fin un vocabulario. Lima: Imprenta Calle de la Encarnación.

Frezier, A. 1902 [1716]. Relación del viaje por el mar del sur a las costas de Chile i el Perú durante los años 1712, 1713 y 1714. Santiago: Imprenta Mejía.

García Óñez de Loyola, M. 1960 [1593]. Ordenanza que deben guardar los administradores de los bienes de los pueblos de indios, dictada por el gobernador Martín García de Oñez y Loyola, 4 de febrero de 1593. In Colección de documentos inéditos para la historia de Chile, segunda serie, vol. IV, pp. 259-267. Santiago: Fondo J. T. Medina.

GonzÁlez De Nájera, A. 1889 [1614]. Desengaño y reparo de la guerra del Reino de Chile. Santiago: Imprenta Ercilla.

Guaman Poma de Ayala, F. 2013 [1615]. El primer nueva corónica y buen gobierno. Mexico City: Siglo XxI.

Guevara, T. 1908. Psicología del pueblo araucano. Santiago: Imprenta Cervantes.

Guevara, T. 1925. Chile prehispánico. Santiago: Universidad de Chile.

Guevara, T. 1928. Sobre el origen de los araucanos. Revista Chilena de Historia y Geográfica 63: 128-168.

Gusinde, M. 1916. Medicina e higiene de los antiguos araucanos. Publicaciones del Museo de Etnología y Antropología 1: 87-121.

IrIBARREN, J. \& Wissing, H. B. 1972. El Camino del Inca en un sector del Norte Chico: una mina de explotación incaica. El Salvador: Talleres del Departamento de Comunicaciones, Compañía de Cobre Salvador.

JAFFUEL, F. 1930. Las piedras pintadas del cajón de Los Cipreses (Hoya del Cachapoal). Revista Chilena de Historia Natural 34 (1): 235-248.

LATCham, R. 1908. ¿Hasta dónde alcanzó el dominio de los incas en Chile? Revista Chilena de Historia Natural 12 (4): 178-199.

LATCHAM, R. 1924. La organización social y creencias religiosas de los antiguos araucanos. Publicaciones del Museo de Etnología y Antropología 3: 245-868.

LAURencich Minelli, L. 2016. La escritura de los incas a la luz de dos documentos de dos jesuíticos secretos recién descubiertos. IHS-Antiguos Jesuitas en Iberoamérica 4 (1): 68-89.

LENZ, R. 1895-1897. Estudios araucanos. Santiago: Imprenta Cervantes.

Llagostera, A. 1976. Hipótesis sobre la expansión incaica en la vertiente occidental de los Andes meridionales. Anales de la Universidad del Norte 10: 203-218.

LolAs E., J. 2018. Imágenes que cuentan la historia del mundo andino. Mensaje 675: 46. 
Martínez, J. L. 1998. Pueblos del chañar y el algarrobo: los atacamas en el siglo XVII. Santiago: DIBAM.

Matienzo, J. DE. 1967 [1567]. Gobierno del Perú con todas las cosas pertenecientes a él y a su historia (1567). Lima: Institut Français d'Études Andines. <https://doi.org/https://doi. org/10.4000/books.ifea.3104> [consutado: 17-03-2021].

Medina, J. T. 1882. Los aboríjenes de Chile. Santiago: Gutenberg.

Miranda Rivera, P. 1958. Quipus y jeroglifos. Zeitschrift für Ethnologie 83 (1): 118-132.

Moesbach, E. DE. 1930. Vida y costumbres de los indígenas araucanos en la segunda mitad del siglo XIX. Santiago: Imprenta Cervantes.

Molina, J. I. 1795. Compendio de la historia civil del reyno de Chile, parte segunda. Madrid: Imprenta de Sancha.

Moulian, R. \& CATrileo, M. 2013. Kamaska, kamarikun y müchulla: préstamos lingüísticos y encrucijadas de sentido en el espacio centro y sur andino. Alpha 37: 249-263.

Moulian, R., Catrileo, M. \& Hasler, F. 2018. Correlatos en las constelaciones semióticas del sol y de la luna en las áreas centro y sur andinas. Boletín del Museo Chileno de Arte Precolombino 23 (2): 121-141.

Nolan, J. \& Urton, G. 2017. Quipus de Pachacamac. Hacia la estandarización de las convenciones de signos en el Tawantinsuyu. In Pachacamac: el oráculo en el horizonte marino del sol poniente. Lima: Banco de Crédito del Perú.

Núñez de Pineda y Bascuñán, F. 1863 [1673]. Cautiverio feliz y razón de las guerras dilatadas de Chile. Santiago: Imprenta del Ferrocarril.

OBREgón ItURRA, J. 2016 Ms. La impotencia colonial ante las asambleas de guerra indígenas. Pujanza depredadora en el entre-dos hispano-indígena. Chile siglo XVII.

Olivari Ortega, J. 1994. El oro entre los incas. Boletín de Lima XVI (91-96): 205-224.

Ovalle, A. DE. 1646. Histórica relación del Reyno de Chile. Rome: Francisco Caballo.

Pereira-González, L. M. \& Batallas-Berdón, B. 2019. Hacia una perspectiva etnomatemática del quipu incaico como puente entre el sistema contable y la escritura. Revista Latinoamericana de Etnomatemática 12 (2): 62-81.

Pietas, J. 1846 [1729]. Noticias sobre las costumbres de los araucanos (11 de junio de 1729). In Historia física y política de Chile. C. Gay, ed., vol. 1, pp. 486-516. Paris: en casa del autor.

Raffino, R., Iácona, A., Gobbo, D. \& Moralejo, R. 2013. La minería y metalurgia de los inkas del Kollasuyu. In Arqueología histórica argentina. Actas del v Congreso Nacional de Arqueología Histórica, E. Rodríguez L. \& D. Schávelzon, eds., pp. 187-216. Saarbrücken: Editorial Académica Española.

Ramírez, J. 2015. Civilización Caral (supe)-Lima-Perú. Su historia y difusión. In Jornada de técnicas de reparación y conservación del patrimonio, IV Congreso Iberoamericano y XII Jornada de Técnicas de Reparación y Conservación del Patrimonio. La Plata: Laboratorio de Entrenamiento Multidisciplinario para la Investigación Tecnológica (LEMIT).
$<$ https://igital.cic.gba.gob.ar/bitstream/handle/11746/1308/ T1-20.pdf-PDFA.pdf? sequence $=1$ \&isAllowed $=\mathrm{y}>$ [consultado: 25-06-2020].

Rosales, D. DE. 1877-1878 [1674]. Historia general de el reyno de Chile, Flandes indiano. Valparaíso: Imprenta del Mercurio.

Salazar, D., Jiménez, C. \& Corrales, P. 2001. Minería y metalurgia: del cosmos a la tierra, de la tierra al Inka. In Tras la huella del Inka en Chile, Museo Chileno de Arte Precolombino, ed., pp. 60-71. Santiago: Museo Chileno de Arte Precolombino.

Shady, R., López, S. \& NARváEZ, J. 2000. La antigüedad del uso del quipu como escritura: las evidencias de la huaca San Marcos. Boletín del Museo de Arqueología y Antropología de la UNMSM 3 (10): 2-23.

STEVENSON, W. B. 1825. A historical and descriptive narrative of twenty years' residence in South America. London: Hurst, Robinson and Company.

Trivero Rivera, A. 2018. Horizonte cultural mapuche. Desde su formación hasta el tiempo actual. J. Calbucura, ed. Sin ciudad editorial: Ñuke Mapuförlaget. <http://www. mapuche.info/wps_pdf/trivero180304.pdf > [consultado: 17-03-2021].

Uhle, M. 1912. Arqueología Sudamericana. Revista Chilena de Historia y Geografía IV (8): 411-425.

URIBE, M. \& SÁNCHEZ, R. 2016. Los incas en Chile: aportes de la arqueología chilena a la historia del Tawantinsuyo (ca. 1400 a 1536 años DC). In Prehistoria en Chile. Desde sus primeros habitantes hasta los incas, F. Falabella, M. Uribe, L. Sanhueza, C. Aldunate \& J. Hidalgo, eds., pp. 529-72. Santiago: Editorial Universitaria.

VAldivia, L. DE. 1606. Arte y gramática de la lengua que corre por todo el Reyno de Chile, con su vocabulario y confesionario. Lima: Francisco del Canto.

Vega, T., Martínez, M., Piombo, M., Bestard, P., Gelós, M. \& SERó, C. 1996. Profundización de los aspectos estéticos de petroglifos y pictografías de la provincia El Neuquén I Parte. Chungara 28 (1-2): 365-379.

VETTER, L. 2007. El papel de los plateros indígenas en la época colonial temprana del virreinato del Perú. Thesis for the degree of Master in History, Pontificia Universidad Católica del Perú.

VIVAR, J. DE. 1966 [1558]. Crónica y relación copiosa y verdadera de los reynos de Chile. Santiago: Fondo Histórico y Bibliográfico José Toribio Medina-Instituto Geográfico Militar.

Zavala, J. M. \& Dillehay, T. 2010. El 'Estado de Arauco' frente a la conquista española: estructuración sociopolítica y ritual de los araucano-mapuches en los valles nahuelbutanos durante los siglos XVI y xviI. Chungara 42 (2): 433-450.

Zavala, J. M., Dillehay, T. \& Medianero, F. J. 2020. Economía aurífera, caminos y fuertes en la Araucanía (Ngülümapu): en torno a la información de Martín Ruiz de Gamboa. Diálogo Andino 61: 27-39. 